\title{
LA PÊCHE THONIÈRE AU GABON : ENTRE EXPLOITATION ET NÉCESSITÉ D'INDUSTRIALISATION LOCALE
}

\author{
Léandre Edgard NDJAMBOU, Aline-Joëlle LEMBE BEKALE, \\ Léonilde Chancia NYINGUEMA NDONG
}

\begin{abstract}
Résumé
L'exploitation du thon au Gabon fait l'objet d'une pêche sous accords privés ou de partenariats depuis plusieurs décennies. Aujourd'hui, avec la prise en compte du domaine maritime dans le Programme Stratégique Gabon Emergent (PSGE), cadre de référence des politiques publiques depuis 2009, la pêche thonière suscite un intérêt soutenu de la part de l'État. Ce dernier ambitionne de renforcer les contrôles en mer, dans la perspective de développer une filière industrielle locale et de trouver un équilibre durable pour les stocks thoniers. Cet article met exergue la problématique de «l'or bleu » dont la maîtrise constitue une source réelle de protéines animales, de dividendes et de diversification économique. De fait, il est particulièrement question de s'appesantir sur les thons tropicaux dont la production est l'apanage des seuls armateurs étrangers. À partir des documents de référence et des données de terrain, la présente contribution se donne l'ambition d'analyser l'organisation de la filière thonière dans son ensemble, d'identifier les dérives qui s'en suivent, mais aussi, de penser les mécanismes favorables à la mise en place d'une industrie du thon au Gabon.
\end{abstract}

Mots-clés

pêche thonière, pêche industrielle, thons tropicaux, accords de pêche, industrialisation

\begin{abstract}
The exploitation of tuna in Gabon has been the subject of fishing under private or partnership agreements for several decades. Today, with the inclusion of the maritime domain in the Emerging Gabonese Strategic Programme (PSGE), the reference framework for public policies since 2009, tuna fishing is arousing sustained interest on the part of the State. The government's ambition is to strengthen controls at sea, with a view to developing a local industrial sector and finding a sustainable balance for tuna stocks. This article highlights the problem of "blue gold", the control of which is a real source of animal proteins, dividends and economic diversification. In fact, there is a particular need to calm down on tropical tunas, whose production is the sole preserve of foreign shipowners. Based on reference documents and field data, this contribution sets out to analyse the organisation of the tuna industry as a whole, to identify the ensuing drifts, but also to consider the mechanisms favourable to the setting up of a tuna industry in Gabon.
\end{abstract}

Keywords

tuna fishing, industrial fishing, tropical tunas, fishing agreements, industrialization

\section{INTRODUCTION}

La ruée vers les ressources biologiques de la mer au tout début des années 1950 a occasionné la surexploitation des poissons comme l'anchois, le cabillaud, le lieu, le merlan et le merlu dans les océans du Nord (Europe, Canada, Islande, Mer du Japon et de Chine) (FAO, 2005). Depuis les années 1990, les captures des pêches maritimes dans le monde stagnent autour de 93 millions de tonnes alors que la consommation des produits halieutiques ne cesse d'augmenter, passant de 9,9 kg en 1960 à 20,1 kg actuellement (FAO, 2016). La hausse qui en résulte, notamment dans les pays en développement $(5,2 \mathrm{~kg}$ en $1961,17,8 \mathrm{~kg}$ en 2013), n'égale cependant pas la consommation des pays développés $(23 \mathrm{~kg}$ en
2013) (FAO, 2014). Ce qui explique la présence des navires asiatiques et européens sur les côtes africaines dès la fin des années 1950 (FAO, 2005).

En effet, la pêche des thonidés le long des côtes africaines est, depuis plus de soixante ans, le fait d'armateurs étrangers comme le témoigne le déploiement des thoniers dans l'Atlantique oriental au début des années 1960 (Anon, 2004). À partir de 1975, le développement des pêcheries s'est progressivement élargi vers le golfe de Guinée, où les premiers accords de thon entre États africains et non africains ont été signés (Carroz et al., 1983). Aujourd'hui, le Gabon, à l'image de la plupart des pays africains ne s'est pas défait de cette physionomie de la culture thonière. 
D'où la sollicitation de l'expertise internationale sous forme d'accords de pêche au regard des investissements considérables et des moyens techniques hautement sophistiqués qu'implique la pêche au thon.

Nous entendons par pêche thonière, toute activité de cueillette qui vise la production de thon dans un but commercial (Saffache, 2003). Elle concerne les thons tropicaux, parmi lesquels, l'albacore (Thunnus albacares) et le listao ou bonite à ventre rayé (Katsuwonus pelamis) qui constituent de loin les espèces emblématiques avec environ $90 \%$ des prises devant le thon obèse ou patudo (Thunnus obesus) et le thon blanc ou germon (Thunnus alalunga).

L'intérêt accordé à la pêche thonière ces dernières années tient au fait qu'une partie importante de thons tropicaux pêchés dans l'Atlantique provient de la ZEE gabonaise, même si les données de capture varient d'une institution à une autre. La Commission Internationale pour la Conservation des Thonidés de l'Atlantique (ICCAT, 2013) estime la production de thons tropicaux pêchés dans l'Atlantique à plus de 300000 tonnes alors que la Conférence Ministérielle sur la Coopération Halieutique entre les États Africains Riverains de l'océan Atlantique (COMHAFAT, 2013) l'évalue à 400000 t. Ainsi entre 80000 à 120000 t de thons proviennent des eaux gabonaises, soit environ 25 à $30 \%$ des prises qui font alors de l'espace maritime gabonais un site majeur pour l'industrie thonière internationale avec l'exportation totale des captures vers les marchés internationaux (espace européen pour l'essentiel), ce qui prive les populations locales de la ressource.

Si la demande gabonaise en poissons est estimée à 70000 tonnes/an, pour une population d'environ 2 millions d'habitants, soit une consommation moyenne annuelle de $30 \mathrm{~kg} /$ habitant, l'offre en produits halieutiques est juste de 40000 tonnes/an (Union, 2019a). La même source révèle que $40 \%$ de la consommation nationale est compensée par les importations. Cette situation pourrait être remédiée par le débarquement partiel de thons dans les ports gabonais, qui devrait couvrir les besoins nationaux en protéines de poisson.

Fort de ce constat, les actions de l'État visent à prévenir la surpêche du thon en formalisant le suivi des quantités exploitées et la traçabilité des captures pour garantir une sauvegarde des populations de thonidés. Dans ce sens, il est nécessaire d'examiner l'activité thonière de fond en comble afin de mieux redéfinir les conditions d'exercice de cette pêche (notamment la préservation des populations d'espèces connexes décimées en lien avec certaines pratiques de pêche), de répondre aux besoins de l'économie et aux attentes des populations en matière de consommation des produits de la mer.

Dès lors, cet article s'inscrit dans la perspective de durabilité d'une activité de pêche en s'appuyant sur les ressources thonières. Un tel objectif suppose que l'on s'attarde sur les mécanismes mis en œuvre dans la production desdites espèces ; c'està-dire les acteurs, les différents accords de pêche, les méthodes de capture ainsi que les tonnages prélevés. Globalement, il est question d'évoquer l'organisation de la filière thon en s'appuyant sur les dispositifs de production, étant entendu que les retombées de cette activité doivent contribuer au développement du secteur halieutique.

La présente contribution permet d'apprécier les conditions d'exploitation du thon au Gabon en s'appuyant sur une analyse des données de terrain ainsi que sur des rapports officiels (2017) et ceux de l'ONG Sea Shepherd Global (2016 et 2017). L'enjeu majeur étant de parvenir à une meilleure réorganisation de l'activité dans la perspective d'établir un équilibre entre les volumes débarqués et la régénération des stocks de thons. Cela implique donc une renégociation des partenariats et l'amorce d'un processus d'industrialisation locale qui tiennent compte des paramètres de durabilité.

L'article soulève ainsi la problématique de l'exploitation des ressources thonières au Gabon au fil des ans. Ainsi peut-on s'interroger sur la meilleure démarche qui prendrait en compte les préoccupations économiques du Gabon (satisfaction de la demande nationale, industrialisation de la filière) et les nécessités de préservation de la ressource? Alors comment arriver à contrôler les captures de thons pour assurer leur durabilité ? Et surtout comment créer une plus-value autour de l'activité thonière au Gabon? Ce questionnement conduit à formuler l'hypothèse selon laquelle, un contrôle rigoureux des activités de pêche thonière en mer permet d'augmenter l'information sur les prises réelles (Tableau 1 et Figure 1). Cette information plus riche que celles 


\begin{tabular}{|l|l|l|l|l|}
\hline \multirow{3}{*}{ Famille } & \multirow{2}{*}{ Type } & Espèce & Statut \\
\cline { 3 - 5 } & & Nom latin & Nom français & UICN \\
\hline \multirow{4}{*}{ Scombridae } & \multirow{4}{*}{ Thonidés majeurs } & Thunnus alalunga & germon & NT \\
\cline { 3 - 5 } & & Thunnus albacares & albacore & NT \\
\cline { 3 - 5 } & \multirow{4}{*}{ Thonidés mineurs } & obèse & VU \\
\cline { 3 - 5 } & & Thunnus obesus & bonitou & LC \\
\cline { 3 - 5 } & & Auxis rochei & auxide & LC \\
\cline { 3 - 5 } & & Euthynnus alletteratus & thonine commune & LC \\
\cline { 3 - 5 } & Katsuwonus pelanis & bonite/listao & LC \\
\hline
\end{tabular}

Tableau 1. Les différents types de thons prélevés et leur vulnérabilité - Source : Programme des Observateurs de la Pêche Industrielle, saison de pêche 2017. * EN : En danger / VU : Vulnérable / NT : Proche de la surexploitation / LC : Préoccupation mineure. N.B : Le thon est prélevé au large des côtes gabonaises à l'abri des contrôles, car la surveillance de cette pêche est pratiquée seulement depuis 2016, à la faveur de l'Opération Albacore menée par l'État gabonais en partenariat avec l'ONG Sea Shepherd Global. Bien que cette ONG évalue les prises à 35000 t/an entre 2013 et 2016, la ressource est totalement débarquée dans les ports étrangers. Il est donc impossible d'estimer les volumes/quantités de chaque espèce, le prix de vente, les destinations ainsi que les circuits de distribution... D'où le réel manque à gagner pour l'économie gabonaise.

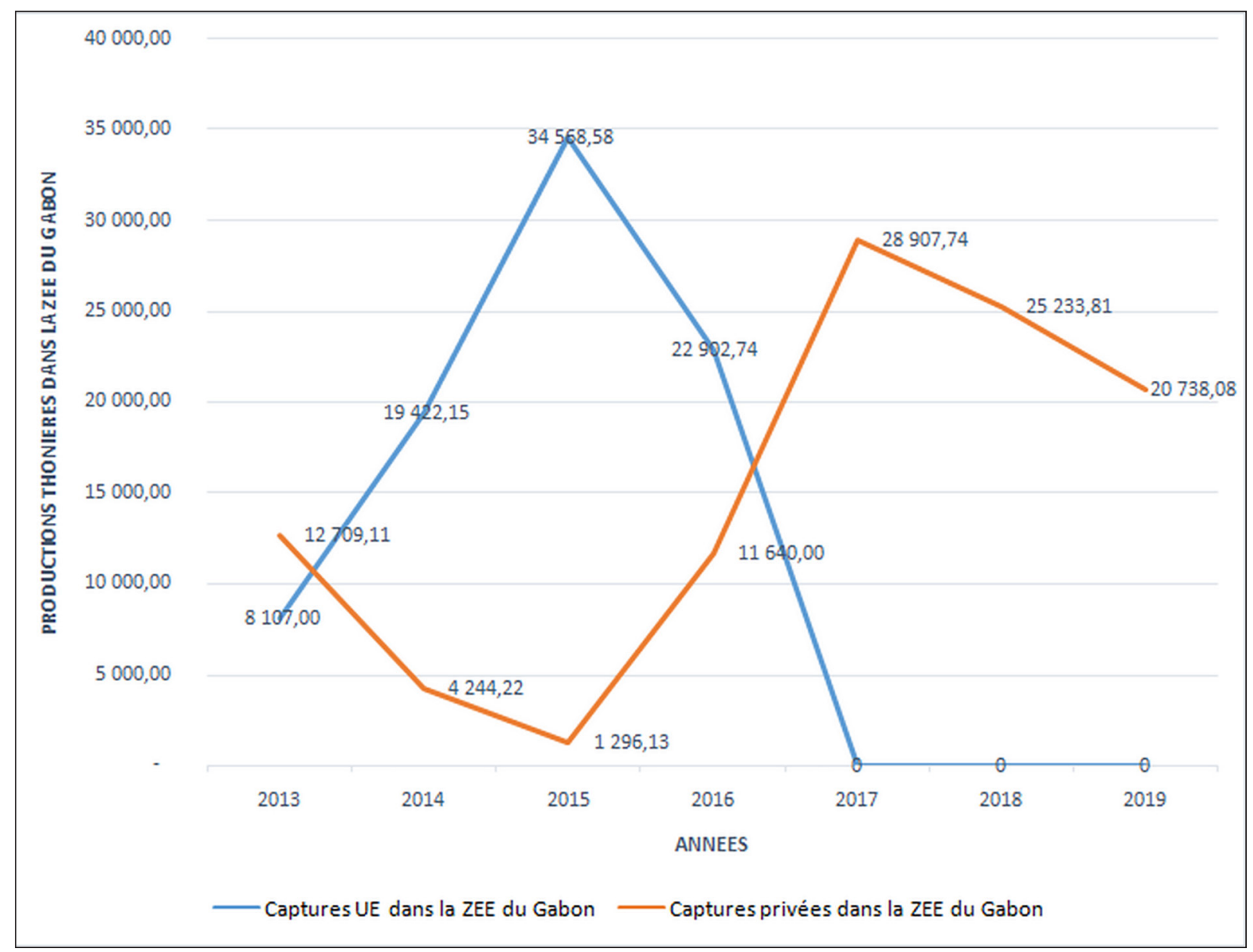

Figure 1. L'évolution de la production thonière dans la ZEE du Gabon - Source : Cellule statistique, DGPA, 2020 
déclarées habituellement par les opérateurs sera une base de discussion pour engendrer un débarquement partiel des captures dans les ports gabonais, d'une part, d'augmenter l'offre en produits halieutiques et de créer une industrie de pêche soucieuse de la pérennité des ressources, d'autre part.

L'analyse s'appuie principalement sur des rapports officiels réalisés dans le cadre du Programme des Observateurs de la Pêche Industrielle (POPI, 2017) à bord des thoniers qui pêchent dans la Zone Economique Exclusive gabonaise (2017) et des données issues des campagnes 2016 et 2017 de l'ONG Sea Shepherd Global sur la pêche illégale au Gabon. De même, l'étude repose sur la consultation des travaux de recherche et des coupures de presse locale (Quotidien l'Union, GabonReview, Gabonactu) qui portent sur différents aspects de la pêche industrielle au Gabon. Enfin, elle se fonde aussi bien sur les observations de terrain que sur les échanges avec les responsables d'administrations en charge du secteur maritime et des activités halieutiques. Les échanges avec les armateurs (Calvo Pesca et Albacora) ont permis de comprendre l'organisation de la filière thonière et les contraintes économiques de leur secteur d'activités. Les rencontres avec les responsables administratifs notamment le Conseil National de la Mer (CNM) et la Direction Générale des Pêches et de l'Aquaculture (DGPA) ont été l'occasion de se faire une idée précise de la vision de l'État gabonais en matière d'industrialisation du thon.

L'article permet de mettre en relief successivement l'écosystème gabonais favorable à la production des thonidés, de décrypter les accords en la matière et de souligner la nécessité de mettre en place une industrie du thon.

\section{LE GABON : UNE PLACE CENTRALE DE PRODUCTION DES THONIDÉS DANS L'OCÉAN ATLANTIQUE}

Le Gabon se présente comme une plaque tournante des pêcheries artisanale et industrielle pour sa diversité et son abondance en ressources halieutiques. Globalement la pêche maritime au Gabon (hors pêche sous accords privés ou de partenariats) reste une activité relativement modeste en termes de production. Celle-ci est estimée à $30000 \mathrm{t}$, soit 20000 t pour la pêche artisanale et 8600 t pour la pêche industrielle côtière (DGPA, 2017).
Parmi les espèces régulièrement prélevées, les thonidés, poissons de luxe, sont particulièrement prisés par l'industrie alimentaire mondiale. Ces ressources à très forte valeur marchande et nutritive apparaissent comme le nouvel or bleu sur lequel compte le Gabon pour impacter son économie maritime et diversifier sa forte ichtyophagie.

\section{A. Des facteurs naturels favorables à la forte ichtyologie thonière}

Au milieu des années 1960, on notait déjà l'importance de la pêche thonière dans le golfe de Guinée notamment dans les environs de l'île d'Annobon (Guinée Equatoriale) située au nord-ouest du Gabon. D'ailleurs, Carré (1998) et Gallardo (1966), faisaient ressortir les caractéristiques physico-chimiques de ce milieu afin de mieux l'appréhender d'une part et d'expliquer ce qui justifiait la présence constante des pélagiques et des thonidés d'autre part. Pour ces derniers, la thermocline influence fortement la présence des espèces pélagiques contrairement aux espèces démersales. Le Gabon, par sa localisation sur l'Équateur apparait comme le point de rencontre de grands courants marins et ces conditions naturelles permettent le développement d'écosystèmes indispensables à la régénération des stocks de poissons (mangroves, coraux), véritables nurseries d'une vaste diversité d'espèces mais d'une grande fragilité. De même, le courant de Benguela qui circule du Cap de Bonne Espérance (Afrique du Sud) jusqu'aux larges des côtes gabonaises est identifiable par un phénomène d'upwelling intense, favorable à la remontée d'eaux froides très riches en nutriments au sud du pays, et dominé par des eaux tropicales chaudes au nord.

Dans le même sens, le renouvellement des eaux est également favorisé par une alternance saisonnière, propice à l'abondance d'une population halieutique en général et des thonidés en particulier. Durant la saison froide (juin à septembre), le système Benguela caractérisé par des eaux froides et salées atteint son intensité. Conjointement, les eaux côtières froides et salées (upwellings) remontent du nord de l'Angola jusqu'au Gabon, dans la zone du Cap Lopez (Goussilou, 2008 ; Berrit, 1961). En saison chaude (janvier à avril), avec la relaxation des upwellings côtiers, le plateau continental gabonais connaît un envahissement par des eaux équatoriales chaudes et moins salées. Simultanément, les eaux froides et salées d'origine tropicale, enrichies par 
le courant de Benguela et les upwellings côtiers arrivent au sud du Cap Lopez. Ce contraste particulier d'eaux froide et chaude constitue à la fois un facteur d'enrichissement, favorable à la reproduction de nombreuses espèces pélagiques (sardine, thon) et joue un rôle déterminant dans la distribution et la concentration de pélagiques hauturiers (thonidés).

\section{B. Une diversité de thonidés prélevés}

Le Gabon est considéré comme un " haut lieu mondial de l'activité thonière » (GabonReview, 2016) au regard de son écosystème maritime riche en diversité de thons, bien que l'imprécision des statistiques ne permette pas d'estimer le volume global disponible dans la ZEE gabonaise. En effet, les sept espèces de thons de la famille des Scombridae prélevées renferment deux types, notamment les thonidés majeurs (grande taille) et les thonidés mineurs (petite taille) (Tableau 1).

Les thonidés majeurs regroupent les thons germon, albacore et obèse. Les thons mineurs concernent quant à eux, la bonitou, l'auxide, la thonine commune et la bonite à ventre rayé ou listao. Au Gabon, les différentes espèces de thonidés sont prélevées dans la ZEE (213 $\left.000 \mathrm{~km}^{2}\right)$ qui correspond à 1,3\% de la surface de l'habitat des thons tropicaux de l'Atlantique (Contrat Cadre Mare, 2015). La pêche au thon se déroule ainsi sur une saison annuelle de cinq mois, de mai à septembre. Elle se pratique uniquement au-delà des douze milles marins à partir des lignes de base et obéit au respect des zones de réserves aquatiques (Carte 1).

La pêche du thon est plus active dans la région du Cap Lopez marquée par une forte saisonnalité et par la présence durant la saison froide du Front du Cap Lopez qui accroît localement la productivité biologique des thons. De fait, de nombreux bancs de thons faciles à localiser et à capturer y sont ainsi concentrés chaque année.

\section{LA PÊCHE THONIÈRE AU GABON : UNE ACTIVITÉ CONTROVERSÉE}

En matière d'exploitation des thonidés, les accords qui lient le Gabon aux pays tiers permettent pour l'heure d'accéder simplement aux ressources inaccessibles aux pêcheurs locaux (absence de technicité) et de contribuer à l'entrée de devises. Mais au-delà de ces deux éléments plausibles, les aspects liés au développement du secteur des pêches et la pérennité des ressources sont loin d'être visibles.

\section{A. La filière thonière gabonaise : une exclusivité d'armateurs étrangers}

Au Gabon, la pêche du thon est pratiquée exclusivement par deux catégories d'armateurs qui interviennent soit dans le cadre d'accords de partenariat, soit dans le cadre d'accords privés. S'agissant de l'accord de partenariat, l'Union européenne a été pendant longtemps l'un des partenaires privilégiés de la pêche du thon au Gabon (Bignoumba, 2007). Cet accord est concrétisé par un protocole triennal. Il définit les principes généraux qui autorisent l'accès des navires de l'UE dans les eaux sous juridiction gabonaise. Il mentionne les modalités et les conditions : montant de redevance, nombre et taille des navires, zones de pêche, types d'engins, captures. Une partie de devises issues de cet accord est dédiée au développement du secteur halieutique essentiellement pour établir un cadre de gouvernance, financer la surveillance de la zone de pêche grâce au suivi par satellite des navires ainsi qu'effectuer des missions de contrôle et d'observation.

Depuis 1998, les deux parties sont liées par un accord autorisant les navires en provenance de l'espace UE de pratiquer la pêche thonière dans les eaux sous juridiction gabonaise. De 1998 jusqu'en fin 2012, cette activité se déroulait sans l'implication de la partie gabonaise, c'est-à-dire sans contrôle des captures. Cependant, le dernier protocole de partenariat Gabon-UE, sur la période 2013-2016, arrivé à échéance et toujours en négociation n'a pas encore été reconduit, notamment pour cause de désaccords relatifs à la clause des Droits de l'Homme d'abord, au tonnage de référence qui impacte la contrepartie financière ensuite, à l'absence d'accompagnement au processus d'industrialisation enfin. Cet accord offrait au Gabon une compensation financière évaluée à 1350000 euros/an dont une enveloppe de 450000 euros qui était principalement orientée vers le renforcement des capacités de l'administration des pêches : acquisition des moyens logistiques, formation des agents, achat d'équipements et logiciels, surveillance des activités de pêche en mer. Ledit protocole permettait l'accès à 27 thoniers avec un tonnage de référence de $10000 \mathrm{t}$ de production maximum par an et par bateau, soit $270000 \mathrm{t}$. Chaque armateur devait s'acquitter de 13750 euros pour obtenir une autorisation de pêche annuelle. 
74 Léandre Edgar NDJAMBOU, Aline-Joëlle LEMBE BEKALE, Léonilde Chancia NYINGUEMA NDONG

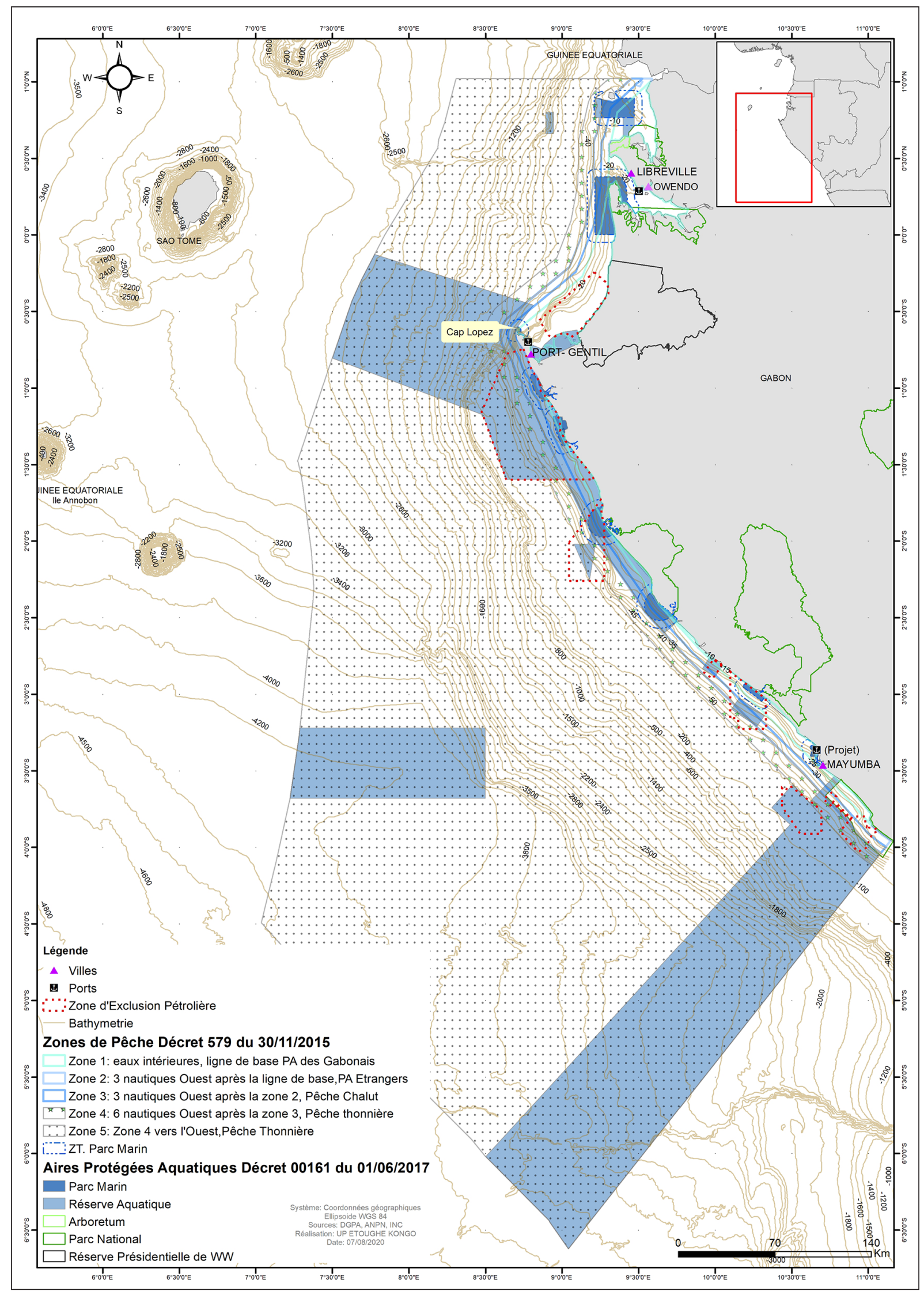

Carte 1. Les activités littorales et maritimes au Gabon 
Par ailleurs, la redevance était acquise en réglant 55 euros par tonne pêchée dès la première année et 65 euros par tonne pêchée les deux années suivantes (UA-BIRA, 2015).

Actuellement, les navires européens ne sont plus autorisés à pêcher dans la ZEE du Gabon. Par conséquent, depuis 2017, l'essentiel de la flotte hauturière est composé d'armements privés. Les accords privés sont conclus entre le Gabon et des entreprises privées immatriculées sous l'État du pavillon ou non. Les accords de pêche conclus par le Japon sont de ce type. Les registres d'immatriculation de la DGPA (2018) indiquent que pour 2017, 18 accords privés de pêche au thon ont été enregistrés (Tableau 2).

La Photo 1 montre la remontée d'une poche de senne enfermant les thons capturés de tailles diverses. La partie inférieure du filet est ramenée vers l'intérieur, tandis que son fond est clos par coulissage ou «boursage», pour éviter aux captures de s'échapper par le bas de l'engin. Néanmoins, des espèces non-ciblées comme les requins, les

\begin{tabular}{|c|c|c|c|c|c|}
\hline Armements & Nom du bateau & Type de pêche & $\mathrm{N}^{\circ}$ de licence & Pavillon & Port d'attache \\
\hline \multirow{14}{*}{ AGAC } & Albacora Nueve & senneur & 25 & Curaçao & Willems Atd \\
\hline & Albacora Caribe & senneur & 26 & Panaméen & Panama \\
\hline & Cape Coral & senneur & 27 & Panaméen & Panama \\
\hline & Sant Yago Très & senneur & 28 & Guatemala & Puerto Quetzal \\
\hline & Sant Yago Uno & senneur & 29 & Guatemala & Puerto Quetzal \\
\hline & Galerna & senneur & 30 & Curaçao & Willems Atd \\
\hline & Montefrisa Nueve & senneur & 31 & Salvador & La Union El S. \\
\hline & Pacific Star & senneur & 32 & Curaçao & Curaçao \\
\hline & Zahara Uno & navire d'appui & 33 & Panaméen & Panama \\
\hline & Guria & senneur & 34 & Curaçao & Willems Atd \\
\hline & Irène & navire d'appui & 35 & Panaméen & Willems Atd \\
\hline & Monteclaro & navire d'appui & 36 & Panaméen & Willems Atd \\
\hline & Montealegre & senneur & 37 & Salvador & La Union El S. \\
\hline & Montecelo & senneur & 38 & Salvador & La Union El S. \\
\hline Sous-total & & & 14 & & \\
\hline \multirow{4}{*}{ ANABAC } & Egalabur & senneur & 39 & Cap-Verdien & San Vicente \\
\hline & Txori Berri & senneur & 40 & Bélisien & Belize City \\
\hline & Playa de Azkorri & senneur & 41 & Bélisien & Belize City \\
\hline & Arene & navire d'appui & 42 & Panaméen & Panama \\
\hline Sous-total & & & 4 & & \\
\hline Total & & & 18 & & \\
\hline
\end{tabular}

Tableau 2. Les navires ayant sollicité une licence de pêche sous accords privés au Gabon en 2017 - Source : ANPA, 2017. Le Tableau 2 montre que la pêche au thon est aujourd'hui le fait des licences privées essentiellement. Ces armements sont également soumis aux mêmes mesures techniques que ceux opérant dans le cadre de l'accord avec l'UE. En effet, 2 principaux armements sous accords privés pêchent le thon au Gabon. Le premier (AGAC) dispose de 14 navires et le deuxième (ANABAC) de 4 navires. Leurs bateaux qui sont très concurrentiels disposent des puissances motrices allant de 2500 à 5000 chevaux (CV) et utilisent des moyens de production modernes et performants. L'ensemble des armements est essentiellement composé de senneurs qui capturent le thon soit sur banc libre (BL), soit par dispositif de concentration de poissons (DCP). La pratique de la senne permet de déployer un filet sur des bancs de thons (adultes et juvéniles) pour prendre de grandes quantités (Photo 1). 
dauphins, les raies et les tortues restent aussi prisonnières. Ces prises qualifiées d'accidentelles sont rejetées dans la mer soit mortes ou vivantes.

Les pêcheurs de thons ont également un fort attrait pour le dispositif de concentration de poissons qui laisse dériver, au gré des courants marins, des objets flottants tels que les radeaux en bois, les vieux cordages pour attirer des bancs de poissons (Photo 2).

La pêche avec des objets flottants naturels (paille, troncs d'arbres) a évolué avec l'introduction de plus en plus d'objets artificiels. Néanmoins, les techniques du Banc Libre et du Dispositif de Concentration des Poissons portent toutes deux atteinte aux stocks disponibles, même si la seconde est constituée par ailleurs d'objets non biodégradables qui occasionnent la pollution des eaux (Tableau 3).

\section{B. Une exploitation thonière au contrôle lâche}

Le contexte actuel d'exploitation du thon au Gabon montre qu'au-delà des compensations financières, les activités dans ce secteur peuvent être remises en question. En effet, la production du thon est de plus en plus critiquée puisqu'elle a du mal à se conformer aux exigences de durabilité. Beaucoup d'armateurs profitent très souvent de l'absence de contrôle et de surveillance du domaine maritime pour mener librement des activités illégales, mieux, pour pratiquer des actes d'incivisme halieutique (Lembe, 2018). Ces activités illégales se manifestent ici par l'absence de sélectivité des captures, le comportement inadapté des équipages et l'absence de transparence d'informations fournies (Tableau 3).

Le Tableau 3 montre les menaces liées à la pêche des thonidés. L'absence de sélectivité est le résultat de l'utilisation massive des DCP au détriment des écosystèmes. Ces techniques de pêche qui ciblent des bancs entiers de thons (espèces matures et juvéniles) compromettent à terme la durabilité des stocks. Aujourd'hui, les principales espèces de thon telles que l'albacore, la bonite à ventre rayé et le thon obèse présentent des stocks vulnérables ou proches de la surexploitation (Tableau 1).

À cela s'ajoute le comportement inadapté des équipages qui manquent de maîtrise dans la mani-

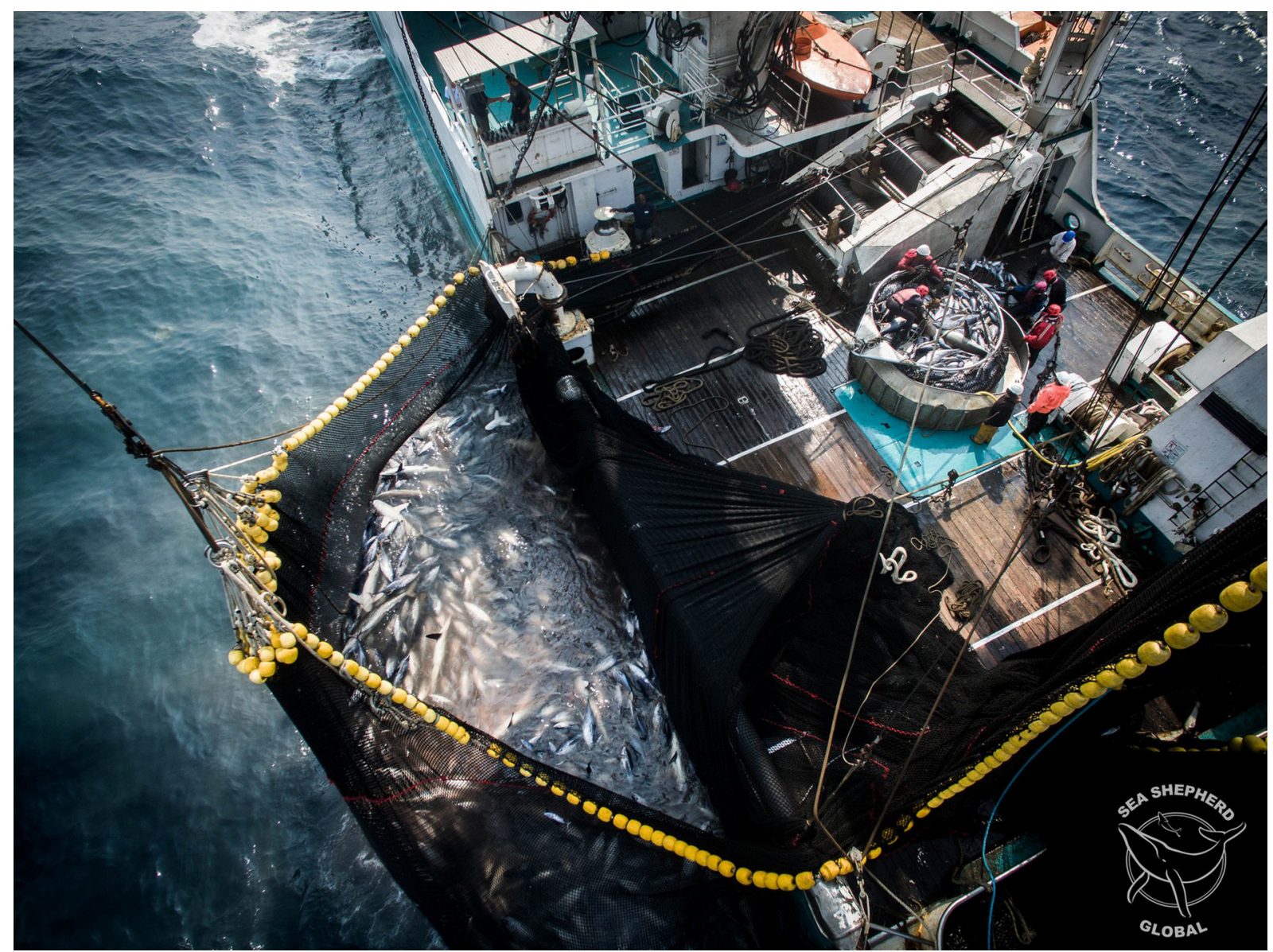

Photo 1. Remontée d'une senne tournante - Source : Programme des Observateurs de la Pêche Industrielle, 2017 


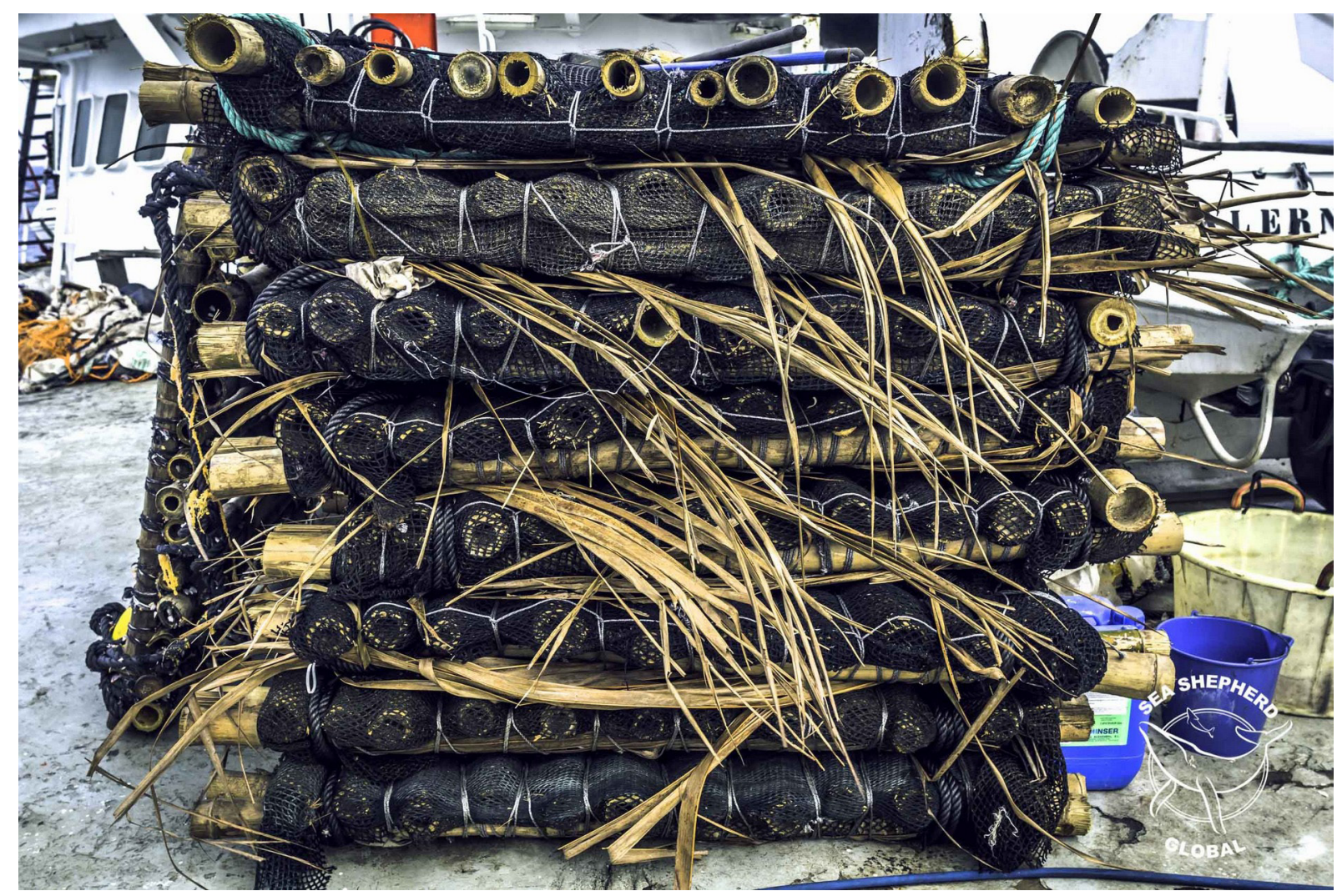

Photo 2. Exemple de DCP en bois - Source : Programme des Observateurs de la Pêche Industrielle, 2017

\begin{tabular}{|l|l|l|}
\hline Menaces & Impacts & Défis \\
\hline $\begin{array}{l}\text { Absence de sélectivité des } \\
\text { captures } \\
\text { (utilisation massive des } \\
\text { DCP) }\end{array}$ & $\begin{array}{l}\text { - Effondrement des stocks } \\
\text { (capture d'adultes matures et } \\
\text { juvéniles immatures) } \\
\text { - Perturbation des migrations } \\
\text { - Pollution par abandon des DCP }\end{array}$ & $\begin{array}{l}\text { - Interdiction des DCP sur banc } \\
\text { libre } \\
\text { - Obligation de récupérer les DCP } \\
\text { en fin de saison }\end{array}$ \\
\hline $\begin{array}{l}\text { Comportement inadapté } \\
\text { des équipages }\end{array}$ & $\begin{array}{l}\text { - Mortalité des espèces sensibles } \\
\text { par mauvais traitement } \\
\text { - Pollution fréquente (dégazage, } \\
\text { DCP ...) }\end{array}$ & $\begin{array}{l}\text { - Formation des équipages sur le } \\
\text { traitement des captures } \\
\text { - Interdiction de la pollution }\end{array}$ \\
\hline $\begin{array}{l}\text { Absence de transparence } \\
\text { (réticence des armateurs) }\end{array}$ & $\begin{array}{l}\text {-Dissimulation d'informations } \\
\text { - Sous-estimation du potentiel } \\
\text { économique de la pêche au Gabon }\end{array}$ & $\begin{array}{l}\text { - Présence obligatoire } \\
\text { d'observateurs à bord des navires } \\
\text { - Suivi régulier des } \\
\text { débarquements } \\
\text { - Débarquement et } \\
\text { transformation au Gabon }\end{array}$ \\
\hline
\end{tabular}

Tableau 3. Récapitulatif des problèmes et défis dans la pêcherie thonière gabonaise - Source : Programme des Observateurs de la Pêche Industrielle, 2017

pulation des captures accidentelles et accessoires. Les taux remarquables de rejets, estimés entre 15 et $20 \%$ selon le rapport des observateurs lors de la campagne 2017, concernent les thons immatures, les espèces sensibles telles que les requins, les tortues et d'autres espèces non valorisées (carangue, baliste...). Ces espèces souvent rejetées dans un état de traumatisme, comateux ou mort témoigne d'une atteinte à la biodiversité et génère par moment des pollutions.

Enfin, le défaut de transparence résulte de la sous-estimation volontaire des captures par les armateurs véreux qui dissimulent très souvent les 
quantités réellement pêchées. Une situation qui est consécutive à l'absence d'experts gabonais à bord des thoniers. Et, même lorsqu'ils sont présents, les experts sont sujets à des tentatives de corruption et de violence. Ce fut le cas, au moment de la campagne de 2017 où des observateurs embarqués sur les navires Sant Yago Tres et Guria, et Sant Yago Tres et Alvarez ont été exposés à des actes d'agressivité, de menaces et refus d'accès aux informations (filmer toutes les manœuvres visant à dissimuler les captures notamment).

Pourtant depuis 2013, le Gabon milite pour un dispositif de contrôle et de surveillance de la pêcherie thonière à travers la présence d'observateurs locaux à bord des thoniers. Ce challenge s'est concrétisé en 2016 avec l'opération Albacore, destinée à assurer le suivi, le contrôle et la surveillance des activités de pêche du thon au large des côtes gabonaises.

Cette démarche a permis de dévoiler les supercheries dans la pêche thonière entre 1998 et 2013. Car, les captures de thons estimées à 7000 t/an sur les $10000 \mathrm{t}$ possibles étaient largement en dessous des productions réelles, puisque dans les mêmes conditions de pêche, les prises sont passées à 35000 tonnes à partir de 2015 (figure 1). Les volumes de thons ont alors été quintuplés grâce à la présence régulière d'observateurs à bord des thoniers. La période allant de 2017 à 2019 correspond à la suspension de l'accord de pêche au thon entre le Gabon et l'Union européenne.

Ces tonnages importants hissent le Gabon au premier rang des producteurs de thons avec $44 \%$ des captures totales de l'océan Atlantique Oriental, devant l'Angola (9,4\%), la Guinée Equatoriale $(6,6 \%)$, la Côte d'Ivoire (3,2 \%), la Sierra Leone $(2,6 \%)$, la Mauritanie $(1,9 \%)$ et le Ghana $(1,2 \%)$ (UA-BIRA, 2015). Cependant, l'exploitation $\mathrm{du}$ thon se traduit par de très faibles retombées socio-économiques pour le Gabon. D'où la nécessité d'envisager une entame de la transformation, au niveau national, de la ressource prélevée, car les captures de thons dans les eaux gabonaises sont de loin les plus importantes et le marché international de conserves de thons est tout aussi porteur. En revanche, la pêche artisanale, essentiellement côtière et estimée à 20000 t/an ne permet pas de couvrir la demande nationale évaluée à 70000 t/an.

\section{DE LA SIMPLE EXPLOITATION À LA NÉCESSAIRE INDUSTRIALISATION LOCALE}

Selon la Commission Internationale pour la Conservation des Thonidés de l'Atlantique (ICCAT) ${ }^{1}$, la production thonière gabonaise est estimée à $20 \%$ de l'ensemble des captures de thons dans l'océan Atlantique, soit environ 45000 tonnes par an (Gabonactu, 2018). Bien que ces réalisations fassent du Gabon le premier pool d'approvisionnement de l'Union européenne, le pays ne dispose pas à ce jour d'infrastructures spécifiquement dédiées au débarquement des thoniers. Il en résulte alors une exportation des prises vers le marché local (débarquement des captures de thons gabonais dans les ports d'Afrique de l'Ouest) et le marché international, annihilant de ce fait la transformation au Gabon de la ressource nationale.

\section{A. L'intérêt d'aménager des infrastructures d'accueil des navires thoniers au Gabon}

Le Gabon compte deux ports commerciaux. Celui d'Owendo, situé à $15 \mathrm{~km}$ au sud de Libreville la capitale, est pourvu de deux quais exploités parallèlement par des opérateurs distincts. Le premier quai, mis en service en 1974 est géré par l'entreprise Gabon Port Management (GPM), filiale du Groupe singapourien Portek, désormais propriété du Groupe japonais Mitsui. Il a une plateforme en béton sur pieux de $455 \mathrm{~m}$ de long sur $70 \mathrm{~m}$ de large. En 1986, s'est ajouté un linéaire d'accostage de $150 \mathrm{~m}$, dédié au déchargement d'hydrocarbures liquides et gazeux en provenance de la ville pétrolière de Port-Gentil. Régulièrement envasé, ce quai offre des profondeurs variables : $11 \mathrm{~m}$ après dragage au poste $n^{\circ} 1,6 \mathrm{~m}$ au poste $n^{\circ} 2,11 \mathrm{~m}$ après dragage au poste $n^{\circ} 3$ et $10 \mathrm{~m}$ au poste $n^{\circ} 4$. Ils sont affectés respectivement à l'accostage des porte-conteneurs, des chalutiers, des vraquiers et au trafic d'hydrocarbures. Le nouveau quai est la propriété d'Olam. Il est l'aboutissement du partenariat public-privé qui a permis la participation à l'investissement du Groupe Olam International et Africa Finance Corporation. Ce nouveau quai dit New Owendo International Port (NOIP) est mis en service en mai 2017. Le quai, long de $690 \mathrm{~m}$, jouxte l'appontement commercial initial.

Le port de commerce de Port-Gentil situé dans la région du Cap Lopez a été mis en service en 1980. 
Il mesure $375 \mathrm{~m}$ de long pour deux postes dont la profondeur varie entre 8 et $11 \mathrm{~m}$. Le quai peut recevoir simultanément deux navires de 150 à $200 \mathrm{~m}$ de long. Le quai de pêche, aménagé perpendiculairement au quai de commerce, est doté d'un linéaire de $280 \mathrm{~m}$ pour un tirant d'eau de l'ordre de 5 à $8 \mathrm{~m}$. Outre les chalutiers dont ceux de la Société Amerger Gabon qui ont choisi le site de Port-Gentil comme port d'attache, ledit quai accueille également des navires pétroliers qui acheminent des produits destinés au forage de nombreux sites pétroliers de la région. Enfin, $75 \mathrm{~m}$ de linéaire, maintenus à une côte moyenne de $-2,5 \mathrm{~m}$, sont réservés aux remorqueurs de servitude.

Néanmoins force est de relever que l'engorgement relatif de ces ports liés à l'étroitesse des quais, d'une part, et à la faiblesse des tirants d'eau réservés aux chalutiers, d'autre part, ne permet pas l'accueil des thoniers lors des campagnes de pêche. Faute de structures portuaires adéquates, le débarquement de la totalité des cargaisons de thons pêchés dans les eaux gabonaises a lieu sur le « marché local» ou sur le marché international. Vers le marché local sont convoyées les espèces présentant une moindre valeur commerciale (thons mineurs). Cette capture couramment nommée «faux poissons » ou «melva» est non taxée. Régulièrement débarquées aux ports d'Abidjan et de Dakar notamment, les prises sont déchargées sur des pinasses qui approvisionnent alors les camions ou les mareyeurs en attente. Les espèces qui alimentent le marché local sont principalement la bonite à ventre rayé, la thonine commune, le bonitou, et l'auxide. Le manque à gagner est double pour l'économie gabonaise. D'une part, la vente de ces thons sur les marchés extérieurs lui échappe. D'autre part, ces captures sont non-taxées par les autorités du Gabon. Or, en 2017, les observateurs gabonais embarqués sur les navires thoniers ont estimé que la production à destination du marché local a représenté 28223 tonnes. Vendue au port d'Abidjan entre 0,3 euro et 0,9 euro le kilogramme (soit entre 200 et 600 Francs CFA le kilogramme), la cargaison a représenté une valeur marchande comprise entre 8466900 euros et 25400700 euros, soit un montant variant entre 5,64 milliards et 16,9 milliards de Francs CFA.

Les captures à destination du marché international, nommées «production», sont quant à elles les seules à être taxées. Selon les dispositions prévues par le dernier Protocole conclu entre le Gabon et l’Union européenne, lesdites taxes s'élevaient à 100 euros par tonne pêchée la première année (2014) et 110 euros par tonnes les deux années suivantes (2015 et 2016). De même, le tonnage excédentaire coûtait 120 et 130 euros par tonne respectivement en 2015 et 2016. Elles correspondent aux espèces qui présentent une haute valeur commerciale (thons majeurs et bonites de grande taille). Souvent débarquées aux ports d'Abidjan et de Dakar, les prises sont ensuite transbordées sur des cargos qui partent pour l'Europe. Les espèces concernées ici sont l'albacore, le thon obèse, le germon et la bonite à ventre rayé (POPI, 2017).

\section{B. Le développement d'une industrie de transformation thonière gabonaise}

Selon le Programme des observateurs de Pêche Industrielle, les thonidés majeurs prélevés en 2017 dans la zone économique exclusive gabonaise ont été pour l'essentiel transbordés dans les ports d'Afrique de l'Ouest sur des navires à destination de l'Europe pour y être traités en usine, transformés et conditionnés en boîtes de conserve. Or, les mêmes conserves sont ensuite distribuées chez les grossistes ou les détaillants au Gabon pour être achetées par les consommateurs. Les thons pêchés au Gabon reviennent ainsi sur le territoire national où ils sont vendus dans les magasins à des prix allant de 8 à 15 euros le $\mathrm{kg}$, soit entre 5245 Francs CFA et 9835 Francs CFA. En estimant qu'en 2017, les captures ont représenté 40 $850 \mathrm{t}$ pour les deux espèces (albacore et thon obèse), les Observateurs évaluent la valeur marchande entre 326800 euros et 612750 euros, soit l'équivalent des montants compris entre 214 milliards et 402 milliards de Francs CFA (POPI, 2017).

La prise en compte du potentiel halieutique national, par l'État gabonais, l'amène aujourd'hui à envisager une industrialisation progressive de la filière pêche en général et thonière en particulier. La première étape de cette "nouvelle approche » consistera à susciter un transbordement des navires européens pêchant dans la ZEE gabonaise dans les ports nationaux. Ainsi seront aménagées les commodités relatives à l'accueil des navires et au stockage de la ressource sur les plateformes portuaires. Dans le même temps, le thon mineur qui alimente encore le marché d'Afrique de l'Ouest viendra satisfaire la demande nationale qui s'en trouve privée. La deuxième étape permettra de débarquer progressivement, dans les ports gabonais, un quota de thons majeurs imposé sur l'ensemble 
des captures réalisées dans la Zone Economique Exclusive nationale. Ledit quota sera alors transformé localement pour créer de la valeur ajoutée. Étant donné que le thon est maintenu congelé à bord du navire, la cargaison destinée au marché international ne souffrira d'aucune entrave. L'objectif recherché est de vendre au niveau national ces produits, de renforcer la sécurité alimentaire du pays et de pouvoir disposer de données réelles sur ces captures qui sont souvent sous-estimées ou dissimulées par les équipages et les armateurs (POPI, op.cit.).

Localement les cargaisons pourront être débarquées soit à Libreville ou à Port-Gentil. Dans cette dernière localité, il existe déjà quelques unités de stockage qui sont la propriété de la société Amerger, spécialisée dans la pêche des crustacés. De plus, Port-Gentil qui est située à seulement 12 heures de navigation des zones de pêche, serait un port idéal de transbordement dans la sous-région (l'Union, 2019b).

En revanche, l'intérêt d'un débarquement des cargaisons de thons à Libreville suppose l'exploitation des installations de Gabon Seafood SA qui sont aujourd'hui laissées en veilleuse, même si selon les spécialistes, les turbines et les chambres froides sont toujours opérationnelles. Héritière de l'ancienne Société Industrielle Frigorifique du Gabon (SIFRI$\mathrm{GAB})^{2}$, Gabon Seafood SA est née d'un partenariat public-privé (PPP), d'un montant de 100 millions d'euros conclu entre l'État gabonais (à travers le Fonds Gabonais d'Investissements Stratégiques (FGIS) et le groupe mauricien Ireland Blyth Limited (IBL). L'accord prévoyait entre autres la reprise par Gabon Seafood SA d'une usine à Owendo (au sud de Libreville) vouée à transformer le poisson et à développer une conserverie de thons et sa chaîne de contrôle. De même, il envisageait la création d'autres structures industrielles, le développement d'un secteur de pêche hauturière et l'aménagement d'un chantier naval au Gabon. Cette entente qui faisait du groupe mauricien le principal partenaire du gouvernement gabonais dans l'optique de la création au Gabon d'une industrie des produits de la mer, l'obligeait à investir initialement $60 \%$ dans le démarrage du projet estimé à 25 millions d'euros, alors que la partie gabonaise devait fournir le reste via le FGIS $^{3}$. Malheureusement, pour des raisons non avouées, Gabon Seafood SAn'a jamais démarré ses activités.

\section{CONCLUSION}

En décidant de faire de la pêche industrielle, singulièrement la pêche thonière, un pôle moteur de l'économie nationale, les autorités gabonaises se sont assignées un objectif noble. Néanmoins l'atteinte dudit objectif implique la mise en place d'un certain nombre de préalables. En effet, considérée comme un secteur stratégique de la diversification de l'économie nationale, la pêche du thon devra bénéficier d'une gestion durable fondée sur une connaissance précise de l'état de la ressource et le renforcement de la gouvernance du domaine. Afin de garantir la régénération du potentiel halieutique, favoriser la capture essentiellement d'individus adultes matures et limiter la pression sur la ressource, il est nécessaire de taxer fortement les prises de petites tailles (individus juvéniles). Un cadre réglementaire attractif devra être élaboré pour intéresser les investisseurs privés nationaux et étrangers aux partenariats publics-privés (PPP).

De même, devra être déclinée clairement la politique retenue pour la transformation locale des produits et l'appropriation d'une partie de la valeur ajoutée. Il est aussi essentiel de renforcer le dispositif d'encadrement et de formation et de l'inscrire de manière précise dans le cadre des négociations des accords de pêche avec l'Union européenne notamment. La matérialisation d'une réelle volonté nationale à industrialiser la filière pêche gabonaise passera par la création d'un armement national favorisant l'insertion des gabonais et l'aménagement d'infrastructures adaptées : quais de débarquement, halls de marchés, conservation sous froid mise à disposition des aires aménagées pour l'implantation d'unités industrielles et pour la transformation artisanale (POPI, op.cit.). Il est aussi primordial de rechercher la mise aux normes des futures unités locales de transformation pour permettre au thon gabonais d'accéder aux grands marchés internationaux.

\section{NOTES}

${ }^{1}$ L'ICCAT est une organisation intergouvernementale qui est active depuis 1969 dans le recueil des données, le suivi scientifique et la gestion des ressources de thonidés et autres espèces apparentées dans l'océan Atlantique et les mers adjacentes.

${ }^{2}$ Créée le 27 octobre 2005 et détenue à $49 \%$ par l'État gabonais et $51 \%$ par la société espagnole Technasa, la 
Société Industrielle Frigorifique du Gabon employait environ 500 agents. Elle était spécialisée dans la production de poisson frais et fumé. Confrontée aux difficultés de trésorerie et d'insuffisance d'outil de production, elle n'a pu réaliser les objectifs qui lui ont été assignés : contribuer à la réduction des importations de poissons, favoriser la consommation d'une variété de produits halieutiques et offrir un important potentiel de développement pour filière pêche nationale.

${ }^{3}$ https : //www.ambagabon.ma « Une usine de transformation du thon au Gabon dans 3 semaines !». Publication de l'Ambassade du Gabon au Maroc, vendredi 25 octobre 2013.

\section{BIBLIOGRAPHIE}

Anon, (2004). Session d'évaluation de 2003 du stock d'albacore. Collect. Vol. Sci. Pap. ICCAT, 56(2), 443-527.

Agence Nationale des Pêches et de l'Aquaculture, (2016). Opération Albacore I. Rapport final, 12 p.

Agence Nationale des Pêches et de l'Aquaculture, (2017). Opération Albacore III. Rapport patrouille 3, 11 p.

Berrit, G.R. (1961). Contribution à la connaissance des variations saisonnières dans le golfe de Guinée - Observations de surface le long des lignes de navigation. Cahiers Océanographiques, XIV(9), 633-643.

Bignoumba, G.-S. (2007). Coopération internationale et exploitation des ressources halieutiques au Gabon. Les Cahiers d'Outre-Mer, 240(4), 393-404.

Carré, F. (1998). La pêche sur les côtes de l'Afrique tropicale, du Sénégal au Congo. Les littoraux espace de vie, Sedès, Paris, pp. 297-306.

Carroz, J. \& Savini, M. (1983). Les accords de pêche conclus par les États africains riverains de l'Atlantique. Annuaire Français de Droit International, 29, 674-709.

Conférence Ministérielle sur la Coopération Halieutique entre les États Africains Riverains de l'Océan Atlantique, (2013). Industrie des pêches et de l'aquaculture au Gabon. Rapport n², 73 p.

Contrat Cadre Mare, (2015). Évaluation rétrospective et prospective du protocole de l'accord de partenariat dans le secteur de la pêche entre l'Union européenne et la République gabonaise. Rapport final, Août 2015, 162 p.

Direction Générale des Pêches et de l'Aquaculture, (2018). Rapport d'activité, 17 p.

Direction Générale des Pêches et de l'Aquaculture, (2017). Tableau d'évaluation des productions 2015 2017, DGPA, Libreville.
Gabonactu, (2018). Lancement de l'opération Albacore 3 pour lutter contre la pêche illégale du thon au Gabon. 12 juillet 2018, 1 p.

GabonReview, (2016). http://economie-gabon.com/ peche-au-thon-le-gabon-haut-lieu-mondial-de-lactivite, $\mathrm{n}^{\circ} 30$ juin, consulté le 20 avril 2019.

Gallardo, Y. (1965). Contribution à l'étude du Golfe de Guinée, hydrologie et courants dans la région d'Annobon. Centre ORSTOM, Pointe-Noire (Congo-Brazzaville), pp.277-288.

Goussilou, J.G. (2008). États des lieux sur les petits pélagiques dans les eaux maritimes du Gabon: cas des sardinelles (Sardinella aurita \& Sardinella maderensis), $16 \mathrm{p}$.

FAO, (2016). La situation mondiale des pêches et de l'aquaculture. Rome, FAO, 227 p.

FAO, (2014). La situation mondiale des pêches et de l'aquaculture. Rome, FAO, 275 p.

FAO, (2005). L'état des ressources halieutiques marines mondiales. Document technique, $\mathrm{n}^{\circ} 457$, Rome, $20 \mathrm{p}$.

ICCAT, (2013). Report of the standing committee on research and statistics (SCRS). ICCAT, Madrid, $344 \mathrm{p}$.

Lembe, A.-J. (2018). La pêche maritime dans les États côtiers d'Afrique centrale : l'incivisme halieutique comme frein au développement durable des ressources ichtyologiques? Revue des Sciences Humaines et des Civilisations africaines, décembre 2018, 174-194.

Le Quotidien d'Informations Générales l'Union, (2019a). Le Gouvernement mise sur le débarquement du thon. $\mathrm{n}^{\circ} 12990$ du lundi 8 avril 2019, 43 année, $24 \mathrm{p}$.

Le Quotidien d'Informations Générales l'Union, (2019b). Pêche/Projet de transbordement des thoniers sous licences privée en saison de pêche dans la ZEE du Gabon. N 12 977, 43 ${ }^{\mathrm{ème}}$ année, 24 p.

Programme des Observateurs de Pêche Industrielle, (2017). Bilan des marées réalisé sur la flotte thonière 2017. 68 p.

Publication de l'Ambassade du Gabon au Maroc. (2019), https : //www.ambagabon.ma « Une usine de transformation du thon au Gabon dans 3 semaines ! » 25 octobre 2013, consulté le 26 avril 2019.

Saffache, P. (2003). Dictionnaire de géographie de la mer et des littoraux. Paris, Ibis Rouge Édition, $101 \mathrm{p}$.

Union Africaine - Bureau Interafricain Des Ressources Animales (UA-BIRA), 2015. Revue des accords de pêche passés et présents conclus par certains Etats membres de l'Union Africaine en Afrique de l'ouest et du centre. Rapport AU-IBAR, 76 p. 
Coordonnées des auteurs :

Léandre Edgard NDJAMBOU

Université Omar BONGO

Département de Géographie

Centre d'Études et de Recherche en

Géosciences Politiques et Prospective

(CERGEP)

ndjambou_leandre@yahoo.fr

Aline-Joëlle LEMBE BEKALE

Université Omar BONGO

Département de Géographie

Centre d'Études et de Recherche en

Géosciences Politiques et Prospective

(CERGEP)

alinejo@yahoo.fr

Léonilde Chancia NYINGUEMA NDONG

Université Omar BONGO

Département de Géographie

Centre d'Études et de Recherche en

Géosciences Politiques et Prospective

(CERGEP)

mimichancia@yahoo.fr 